\title{
Identification of FISH biomarkers to detect chromosome abnormalities associated with prostate adenocarcinoma in tumour and field effect environment
}

\author{
Ying Zhang ${ }^{1}$, Thomas Perez ${ }^{1}$, Beth Blondin ${ }^{1}$, Jing Du', Ping Liu', Diana Escarzaga², John S Coon², \\ Larry E Morrison ${ }^{1}$ and Katerina Pestova ${ }^{1 *}$
}

\begin{abstract}
Background: To reduce sampling error associated with cancer detection in prostate needle biopsies, we explored the possibility of using fluorescence in situ hybridisation (FISH) to detect chromosomal abnormalities in the histologically benign prostate tissue from patients with adenocarcinoma of prostate.

Methods: Tumour specimens from 33 radical prostatectomy (RP) cases, histologically benign tissue from 17 of the 33 RP cases, and 26 benign prostatic hyperplasia (BPH) control cases were evaluated with Locus Specific Identifier (LSI) probes MYC (8q24), LPL (8p21.22), and PTEN (10q23), as well as with centromere enumerator probes CEP8, CEP10, and CEP7. A distribution of FISH signals in the tumour and histologically benign adjacent tissue was compared to that in BPH specimens using receiver operating characteristic curve analysis.
\end{abstract}

Results: The combination of MYC gain, CEP8 Abnormal, PTEN loss or chromosome 7 aneusomy was positive in the tumour area of all of the 33 specimens from patients with adenocarcinomas, and in $88 \%$ of adjacent histologically benign regions (15 out of 17) but in only 15\% (4 out of 26) of the benign prostatic hyperplasia control specimens.

Conclusions: A panel of FISH markers may allow detection of genomic abnormalities that associate with adenocarcinoma in the field adjacent to and surrounding the tumour, and thus could potentially indicate the presence of cancer in the specimen even if the cancer focus itself was missed by biopsy and histology review.

Keywords: Prostate cancer, Genomic abnormalities, Diagnosis, Field effect, Fish, Fluorescence in situ hybridisation

\section{Background}

Prostate carcinoma is the most common type of cancer in men in the United States, with an estimated 241,740 new cases in 2012 and 28,170 deaths [1]. It is the second leading cause of cancer death in US men after lung cancer. The absence of reliable diagnostic markers that enable early and accurate detection of carcinomas when they are confined to the prostate is a fundamental problem in the management of prostate cancer. The leading early detection and diagnostic approach employs a combination of DRE (digital rectal examination) and measurement of serum PSA (prostate-specific antigen) followed by a

\footnotetext{
* Correspondence: ekaterina.pestova@abbott.com

'Abbott Molecular, Inc, 1300 East Touhy Avenue, Des Plaines, IL 60018, USA Full list of author information is available at the end of the article
}

prostate biopsy. However, this approach has major limitations. Out of the approximately 1.2 million patients who undergo prostate biopsy each year in the US, $70 \%$ to $80 \%$ receive negative results [2]. These patients cannot be completely reassured, however, because a cancer might have been missed by sampling error due to the focal nature of Prostate cancer $(\mathrm{CaP})[3,4]$. Therefore, each year about 840,000 to 960,000 men undergo repeat biopsies because of consistently elevated PSA levels [4]. An additional challenge in prostate cancer diagnosis is that prostate cancer is a multi-focal disease, with $67 \%$ to $96 \%$ of radical prostatectomy specimens containing more than one focus of disease [5-7]. Studies have shown that the use of more biopsy cores may improve accuracy of diagnosis, reducing the sampling effect [8]. Currently, a 12-core scheme is

\section{()


recommended as a reasonable biopsy approach, providing an acceptable sampling of the prostate gland [9], however, collection of a higher number of biopsy cores is also being considered [2]. Due to the potential comorbidity associated with collection of the high number of biopsy cores, utilisation of molecular assays to improve the diagnostic accuracy would be beneficial. Combined with conventional histopathological assessment, predictive biomarkers that indicate the high likelihood of the presence of malignancy on a biopsy specimen without clearly malignant histology could provide clinicians with guidance for stratifying individuals into those who need repeat biopsy or intensive follow-up and those who do not.

The concept of "field cancerisation" or "field effect" was first proposed by Slaughter et al. in 1953 when observing histological features of oral cancers [10] to describe the presence of histologically abnormal tissue surrounding primary cancerous lesions. It was considered to cause the occurrence of multifocal tumours and cancer recurrence. Due to the tremendous progress in molecular biology and biotechnology, the definition of field effect has been extended to the molecular abnormalities in tissues that appear histologically benign, as defined by Höckel and Dornhöfer [11]: "the monoclonal or multiclonal displacement of normal epithelium by a genetically altered but microscopically undistinguishable homologue." Since then, the presence of the field effect has been reported in various tumour types, including carcinoma of the head and neck, lung, colon and rectum, breast, stomach, prostate, and urinary bladder $[12,13]$.

Prostate cancer is multifocal disease, and field effect may play a fundamental role in the development of multifocal lesions. A recent review by Trujillo KA et.al [14] summarized that field cancerisation of prostate can occur at the levels of genetic, epigenetic, and biochemical aberrations in structurally intact cells in histologically normal tissues adjacent to cancerous lesions. Prostate cancer biomarkers of field cancerisation have been studied by several groups using different strategies, including nuclear morphology, DNA methylation, Mitochondrial DNA changes, mRNA profiling, protein expression and genomic DNA changes [14]. Genomic, epigenetic, and biochemical alterations observed outside the histologically visible tumour margins could result from pre-existing fields of precursor cells in which cancer develops; alternatively, the tumour could have an effect on the surrounding tissue, or the observed abnormalities could reflect both of the above effects [15]. The question whether the field of molecular alterations is exclusively of precursor nature, or whether it is induced by the tumour, is still being discussed in the literature [14]. However, irrespective of their origin, the markers of field cancerisation are associated with the cancer, and could indicate the presence of cancer in the specimen if detected in the tumour-adjacent histologically benign tissue.

Although Florescence In Situ Hybridization technique (FISH) represents a molecular technique that allows the detection of numerical and structural genomic abnormalities in interphase cell nuclei in tissue sections or cytological specimens such as deletion, amplification, and translocation of various genomic regions in many types of cancer [16,17], it has not been widely used in the studies on field cancerisation. Multiple chromosomal alterations have been reported in $\mathrm{CaP}$ [18-20], including chromosome aneusomy, gain of the 8q24 (MYC) locus, and loss of 8p21-22 (LPL) [21], and 10q23 (PTEN), among others [22]. In our initial feasibility study, we evaluated aberrations in multiple genomic loci involved in tumorigenesis by FISH on a set of FFPE prostate adenocarcinoma specimens, and selected a group of probes that detected cytogenetic abnormalities in these tumours. The selected probes included $L P L, M Y C$, PTEN, CEP7, CEP 8, and CEP 10. In this study, using FISH technique, we assessed whether these biomarkers could detect chromosomal abnormalities that are present in the histologically benign region adjacent to frank carcinoma.

\section{Methods}

\section{FISH probes}

A total of 6 probes including 3 centromeric probes $\left(\mathrm{CEP}^{\circ}\right)$ and 3 locus-specific identifiers $\left(\mathrm{LSI}^{\circ}\right)$ were used. CEP probes included CEP7 (SpectrumAqua ${ }^{\mathrm{Tu}}$ ), CEP8 (SpectrumAqua), and CEP10 (SpectrumGreen ${ }^{\text {Tw}}$ ). LSI probes were PTEN (SpectrumOrange ${ }^{\mathrm{Tx}}$ ), MYC 8q24 (SpectrumGreen), and LPL 8p21-22 (SpectrumOrange). All probes were obtained from Abbott Molecular, Inc. (Des Plaines, IL).

\section{Histological specimen collection}

Thirty-three archived RP cases from patients with prostate adenocarcinoma and 26 control Benign Prostatic Hyperplasia (BPH) cases were provided by Rush University Medical Center (Approved IRB L06052503 waived the requirement for informed consent). Multiple tissue blocks were prepared from each of the RP cases. For each specimen, $5 \mu \mathrm{m}$ tissue sections were cut and placed on positively charged microscope slides. The blocks were characterised by staining one out of 10 serial sections through the block with haematoxylin and eosin (H\&E) followed by examination by an expert pathologist. For all 33 cases, at least one block containing adenocarcinoma was identified for this study and designated as "tumour". Region(s) with histopathological features of adenocarcinoma were marked by the pathologist on the H\&E slides of the tumour specimens. Twenty-five of the 33 adenocarcinoma cases were determined to have a Gleason 
score of 5-7, 6 cases had a Gleason score of $>7$, and 2 had a Gleason score of 2-4. For 17 out of 33 adenocarcinoma cases used in this study, a second block was identified that contained no recognisable histological features of adenocarcinoma or prostatic intraepithelial neoplasia (PIN) and designated as "histologically benign." It was estimated that histologically benign tissue was spatially separated from the tumour margin on average by approximately $1 \mathrm{~cm}$. The H\&E images of the 17 histologically benign slides used in the study are provided in the Additional file 1. For the BPH cases, FFPE blocks of TURP specimens were utilized. For each TURP specimen, $5 \mu \mathrm{m}$ tissue sections were cut and placed on positively charged microscope slides. The blocks were characterised by staining one out of 10 serial sections through the block with haematoxylin and eosin (H\&E) followed by examination by an expert pathologist to confirm that no histological features of adenocarcinoma or prostatic intraepithelial neoplasia (PIN) are present.

The specimen slides used for the FISH assay procedure were within 10 serial sections of the respective H\&E-stained slide to assure minimal separation of the areas examined by FISH from the areas evaluated by histopathology.

\section{Histological sample pre-treatment and hybridisation}

Formalin fixed paraffin embedded (FFPE) histological specimen slides were baked at $56^{\circ} \mathrm{C}$ for $2-24$ hours, then were treated three times in Hemo-De (Scientific Safety Solvents) for 5 minutes each at room temperature followed by two 1-minute rinses in $100 \%$ ethanol at room temperature. Slides were incubated in pre-treatment solution $(1 \times \mathrm{SSC}, \mathrm{pH} 7.0)$ at $80^{\circ} \mathrm{C}$ for 35 minutes, rinsed for 3 minutes in deionized water, incubated $20-22$ minutes in $0.15 \%$ pepsin in $0.1 \mathrm{~N} \mathrm{HCl}$ solution at $37^{\circ} \mathrm{C}$, and rinsed again for 3 minutes in deionized water. Slides then were dehydrated for 1 minute each in 70\%, 85\%, and 100\% ethanol and air-dried. Two sets of probe hybridisation mix were made: Probe mix 1 included CEP8 (SpectrumAqua), MYC 8q24 (SpectrumGreen), and LPL 8p21-22 (SpectrumOrange); Probe mix 2 consisted of CEP7 (SpectrumAqua), CEP10 (SpectrumGreen), and PTEN (SpectrumOrange). Ten microliters of either probe hybridisation mix containing blocking DNAs and LSI/WCP Hybridisation Buffer (Abbott Molecular, Inc., Des Plaines, IL) were added to a specimen, and a coverslip was applied with rubber cement sealed around. Slides and probes were codenatured for 5 minutes at $73^{\circ} \mathrm{C}$ and hybridized for $16-24$ hours at $37^{\circ} \mathrm{C}$ on a ThermoBrite hybridisation platform (Abbott Molecular, Inc.). Following hybridisation, coverslips were removed by soaking the slides in $2 \times \mathrm{SSC} / 0.3 \% \mathrm{NP}-40$ for $2-5$ minutes, and immediately slides were washed in $2 \times \mathrm{SSC} / 0.3 \% \mathrm{NP}-40$ at $73^{\circ} \mathrm{C}$ for 2 minutes and subsequently in $1 \times$ SSC solution,
$\mathrm{PH} \sim 7.0$ for 1 minute at room temperature. The slides were then allowed to dry in the dark. Ten microliters of 4',6-diamidino-2-phenylindole counterstain/antifade solution (DAPI I, Abbott Molecular, Des Plaines, IL) was added to the specimen, and a coverslip was placed on the slide for microscopy.

\section{FISH signal evaluation}

The specimens were analysed under a fluorescence microscope using single bandpass filters (Abbott Molecular, Des Plaines, IL) specific for DAPI, SpectrumOrange, SpectrumGreen, and SpectrumAqua. The number of FISH signals for each probe was recorded in a minimum of 50 consecutive non-overlapped, intact interphase nuclei in areas of interest, which were identified by DAPI staining of nuclei with reference to the corresponding H\&E-stained tissue. Tumour areas (tumour regions of interest, ROI) scribed by the pathologist were evaluated on the 33 RP specimen slides. For the 17 slides with histologically benign tissue adjacent to tumour, representative areas were evaluated (histologically benign regions of interest, ROI). Similarly, representative areas were evaluated on $\mathrm{BPH}$ specimen slides.

\section{Statistical analysis}

For each specimen, 50-100 cells were enumerated with respect to the number of fluorescent signals of each probe. The following FISH abnormality parameters were calculated for each probe:

- \%Gain, percent cells with $>2$ signals

- \%Loss, percent cells with $<2$ signals

- \%Abnormal, percent cells with $>2$ or $<2$ signals

- For the two probe ratios (probe A/probe B), \%Gain is the percentage of cells with $A / B$ ratio $>1$, and \%Loss is the percentage of cells with $A / B$ ratio $<1$.

In order to screen for FISH probes potentially important for disease detection, the FISH parameters described above were compared between different specimen groups (tumour ROI vs. $\mathrm{BPH}$, and histologically benign ROI vs. BPH) using a two-sample $t$-test. FISH parameters with significant $\mathrm{p}$-values $(\mathrm{p}$-value $<0.05)$ from the $t$-test were selected for further examination.

After prioritizing potential FISH probes, the receiver operating characteristic (ROC) method [23] was used to select optimal FISH probe combinations, as well as the optimal cut-off value for individual FISH probes. The ROC curve is a plot of sensitivity versus 1 -specificity or false positive rate (FPR). In our study, each point on the ROC curve represents a sensitivity/specificity pair corresponding to a particular cut-off (for single FISH parameter) or a combination of cut-offs (for FISH parameter 
combinations). From the ROC curves, the distance from ideal (DFI) and the area under the curve (AUC) were calculated.

DFI is defined as $\sqrt{(1-\text { sensitivity })^{2}+(1-\text { specificity })^{2}}$. DFI represents the minimum distance from the ROC curve to the value of a sensitivity of 1 and a false positive rate (1-specificity) of 0 . The DFI ranges from 0 to 1 , with 0 being the ideal. Determination of the "optimal" cut-off value is always a trade-off between sensitivity and specificity. Ideally, the "optimal" cut-off value provides both the highest sensitivity and the highest specificity, easily located on the ROC curve by finding the point with minimum DFI. For determination of optimal cut-off value, minimal DFI was used as the selection criterion. The parameters with the determined cut-off below the level of truncation for the FISH signals in FFPE tissue specimens were not included in the further analysis. For determination of optimal FISH probe combination, AUC was used as the selection criterion. Statistically, the best FISH probe combination is the one with largest AUC values. For the selection of the final, optimized probe set, scientific judgment was applied in combination with the statistical analysis.

All analyses were performed using SAS version 9.2 (SAS Institute Inc., Cary, NC, USA.) on a UNIX operating system by Abbott Molecular Biostatistics and Data Management Group.

\section{Results}

\section{Detection of cytogenetic abnormalities by FISH in RP specimens}

In an initial feasibility study, we tested 16 RP specimens from patients with adenocarcinoma of the prostate. Nine out of the 16 specimens had a second section available with only histologically benign tissue. Slides from $11 \mathrm{BPH}$ cases were used as controls. The probes included LSI MYC (8q24), LPL (8p21.22), PTEN (10q23), and p16 (9p21), centromere probes CEP8, CEP10, CEP7, CEP3, and CEP17, and TMPRSS2 break-apart. In the study, we observed that tumour ROIs in radical prostatectomy specimens bore chromosomal abnormalities, including $M Y C$ amplification/gain, LPL and PTEN loss, TMPRSS2 rearrangement, as well as general aneuploidy. Importantly, we also observed chromosomal abnormalities in some of the 9 histologically benign slides from tumour patients. The results demonstrated the feasibility of using a FISH assay to detect chromosome abnormalities that are specific to specimens from adenocarcinoma patients. Based on these initial results, we selected six probes that detected chromosomal copy number abnormalities in most RP specimens, both within tumour regions and extending beyond histologically evident tumour. The six probes included MYC, LPL, CEP8, PTEN, CEP10, and CEP7.
We expanded the initial specimen set to the total of $33 \mathrm{RP}$ specimens from patients with adenocarcinoma of prostate and $26 \mathrm{BPH}$ control specimens for the interrogation with the six selected FISH probes. For 17 out of 33 adenocarcinoma cases, in addition to the tumour region, we also evaluated tumour-adjacent histologically benign tissue from the same specimen, using a separate FFPE block that contained no histological features of adenocarcinoma or prostatic intraepithelial neoplasia (PIN) upon histopathological examination.

Chromosomal abnormalities of $M Y C, L P L$ and PTEN, and aneusomy (as measured by copy number changes of the chromosome-specific CEP probes) were observed in tumour ROIs of the radical prostatectomy specimens. Figure 1 shows images representing the copy number gain of MYC (Figure 1A and B) and the loss of PTEN (Figure $1 \mathrm{C}$ and $\mathrm{D}$ ). Images were recorded within the tumour ROI, which could also be recognized by the characteristic pattern of nuclei under DAPI staining (Figure $1 \mathrm{~A}$ and $\mathrm{C}$ ). MYC signal was clearly gained (Figure 1B, displaying 3 or 4 signals per cell in this specimen), while PTEN signal was lost (Figure 1D), showing either zero or one copies in the majority of cells within the ROI.

In this study, FISH analysis was performed on histological specimens. In contrast to cytology, FISH on FFPE tissue specimens presents artefacts related to the nuclear truncation. In our study, FISH analysis of individual signal counts showed a loss up to $10 \%$ of signals, with average counts of FISH probe signals per cell of 1.80-1.84 in both test (RP) and control (BPH) tissue specimens. Therefore, although truncation effects were evident, the level of truncation did not appear to differ between test and control cases. To further control for artefacts of nuclear truncation, the cut-offs for FISH positivity, especially for deletion probes, were chosen above the level of truncation, as presented in the section Data Analysis of Probe Performance below.

Sixteen parameters derived from genomic copy numbers detected by FISH were evaluated by the $t$-test comparing tumour ROI and BPH. These parameters were CEP10\% Abnormal, CEP10\%Gain, CEP7\%Abnormal, CEP7\%Gain, CEP8\%Abnormal, CEP8\%Gain, CEP8\%Loss, MYC\%Gain, LPL\%Abnormal, LPL\%Loss, PTEN\%Loss, PTEN/CEP10\% Loss, CEP7/CEP10\%Gain, LPL/CEP8\%Loss, MYC/CEP8\% Gain and $M Y C / L P L \%$ Gain. Results from $t$-test analyses demonstrated that for all of the 16 FISH parameters, mean values were statistically different between tumour and $\mathrm{BPH}$ groups (Additional file 2: Table S2a). Interestingly, chromosomal abnormalities were observed not only in the tumour ROIs, but also on slides with the histologically benign tissue approximately $1 \mathrm{~cm}$ away from the tumour margin of the RP adenocarcinoma specimens (benign ROI).

Representative images demonstrating FISH and $\mathrm{H} \& \mathrm{E}$ staining from a tumour section (Figure 2A, 2B, and 2C) 

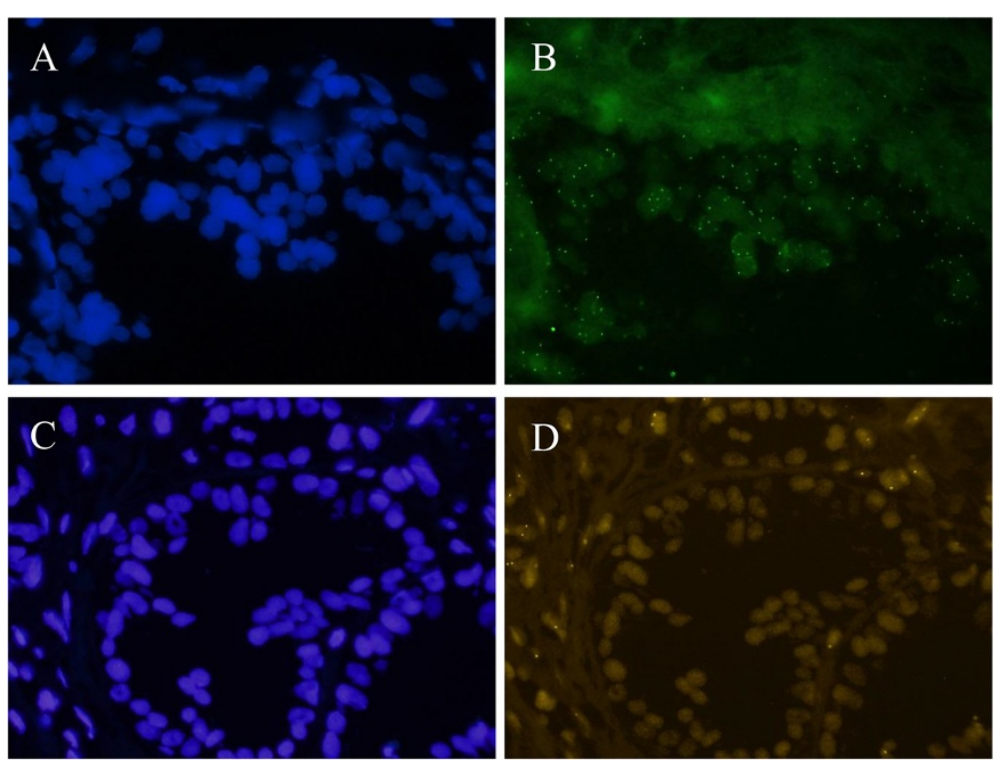

Figure 1 Images of abnormal FISH signals. A and $\mathbf{B}$ : The images of MYC SpectrumGreen copy number gain with DAPI staining (A) showing the nuclei morphology, and MYC staining (B) displaying gain of copy numbers. $\mathbf{C}$ and $\mathbf{D}$ : the images of PTEN loss with DAPI staining (C) of the tumour ROI, and PTEN FISH hybridisation showing deletion of PTEN in the prostate gland (D).

and a histologically benign section (Figure 2D, 2E, and $2 \mathrm{~F}$ ) of the same case are shown in Figure 2. Figure 2A presents an H\&E image of a tumour section (from Case 01, tumour block) with the area of the tumour circled. Figure 2D shows an H\&E image of the histologically benign section (from Case 01, histologically benign block) with the area that had abnormal FISH signals circled. Figure $2 \mathrm{~B}$ and $2 \mathrm{C}$ show abnormal FISH in a representative field of view of the tumour section. Figure $2 \mathrm{E}$ and 2F show abnormal FISH from a representative field of view of the histologically benign section. Figure $2 \mathrm{~B}$ and $2 \mathrm{E}$ show MYC amplification (green signals indicated by the red arrow) and DAPI nuclear staining (blue), while Figure $2 \mathrm{C}$ and $2 \mathrm{~F}$ show PTEN deletion indicated by green arrows (gold PTEN signals are visible only in stroma cells, indicated by white arrows) and DAPI nuclear staining (blue).

To confirm that the histologically benign areas selected for the study were indeed devoid of tumour features, we utilized a second, independent pathologist to review the corresponding H\&E images. Upon the indepth review of the suspected area, all but one specimen were deemed to be histologically normal, since no features of prostate adenocarcinoma, or PIN were observed in these regions. In one apparently benign specimen (sample number 33 block B), a very close, detailed inspection by the independent pathologist revealed possible minute tumour foci, however, the pathologist was not able to conclusively classify the observed cells as tumour without a suggested confirmation by other methods (such as IHC), highlighting the challenges in histopathological assessment of prostate tissue specimens. Therefore, we confirmed that FISH detected cytogenetic abnormalities in the regions of prostate that would not have been identified as tumour on histopathology review.

Results from t-tests comparing histologically benign ROI and BPH controls, showed that for 10 out of the 16 FISH parameters (Additional file 2: Table S2b), mean values were statistically different between the comparison groups. These 10 FISH parameters, derived from copy numbers of MYC, LPL, CEP8, PTEN, CEP10 and CEP7, were CEP10\%Abnormal, CEP10\%Gain, CEP7\%Abnormal, CEP7\%Gain, CEP8\%Abnormal, CEP8\%Gain, MYC\%Gain, LPL\% Abnormal, PTEN\%Loss, PTEN/CEP10\%Loss.

\section{Analysis of probe performance and selection of optimal probe combinations}

The receiver operating characteristic (ROC) analysis method was applied to the selected ten single FISH parameters. AUC values from the ROC analysis comparing histologically benign ROIs from adenocarcinoma RP specimens with BPH controls were used to assess the selected FISH parameters in respect to their ability to distinguished adenocarcinoma specimens from the $\mathrm{BPH}$ controls based on the presence of the genomic abnormalities extending beyond the tumour margin. Table 1 summarizes the sensitivity and specificity of the selected FISH parameters at the optimal cut-off for each parameter. In this analysis, the cut-off was determined based on the shortest Distance From Ideal (DFI) as described in Methods. All of the selected parameters were also 

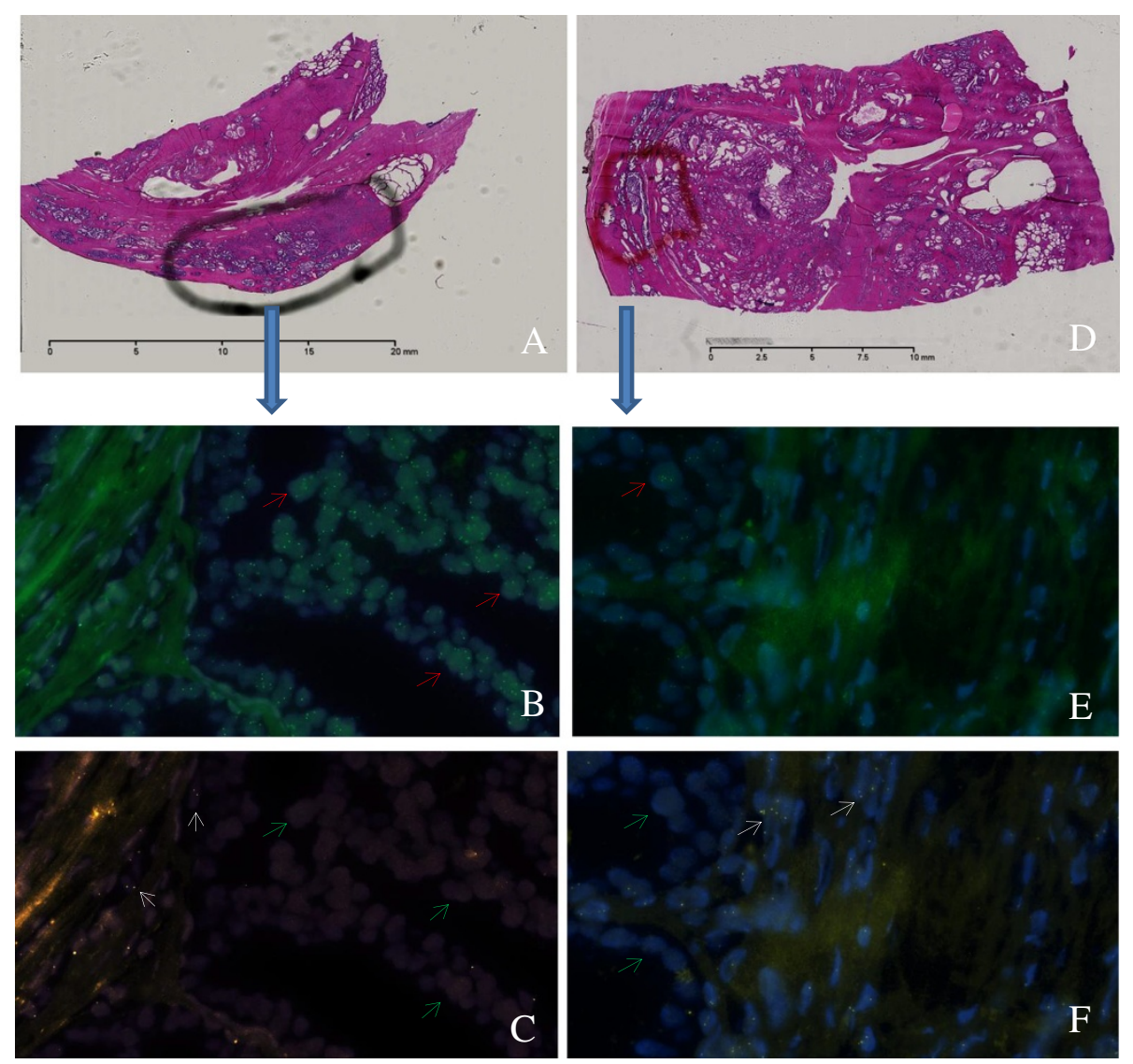

Figure 2 Representative images of FISH and H\&E staining from tumour and histologically benign areas of the same case. A: an H\&E image of a tumour section (from Case 01, tumour block) with the area of tumour circled; B: MYC amplification (green signals indicated by the red arrow) and DAPI nuclear staining (blue) in a representative field of view of the tumour section; C: PTEN deletion indicated by green arrows and DAPI nuclear staining (blue) in a representative field of view of the tumour section (gold PTEN signals are visible only in stroma cells); D: an H\&E image of the histologically benign section (from Case 01, histologically benign block) with the area that has abnormal FISH signals circled; E: MYC amplification (green signals indicated by the red arrow) and DAPI nuclear staining (blue) in a representative field of view of the histologically benign section; F: PTEN deletion indicated by green arrows and DAPI nuclear staining (blue) in a representative field of view of the histologically benign section (gold PTEN signals are visible only in stroma cells, indicated by white arrows).

Table 1 ROC analysis (sensitivity, specificity, and the AUC) comparing ten selected FISH probe parameters

\begin{tabular}{|c|c|c|c|c|c|c|}
\hline \multirow{2}{*}{$\begin{array}{l}\text { FISH probe } \\
\text { parameters }\end{array}$} & \multicolumn{3}{|c|}{ Tumour - BPH } & \multicolumn{3}{|c|}{ Histologically benign - BPH } \\
\hline & Sensitivity & Specificity & AUC & Sensitivity & Specificity & AUC \\
\hline CEP8\%Gain & $79.40 \%$ & $76.90 \%$ & 0.853 & $82.40 \%$ & $76.90 \%$ & 0.871 \\
\hline MYC\%Gain & $91.20 \%$ & $76.90 \%$ & 0.908 & $82.40 \%$ & $76.90 \%$ & 0.846 \\
\hline CEP7\%Abnormal & $88.20 \%$ & $80.80 \%$ & 0.896 & $76.50 \%$ & $80.80 \%$ & 0.845 \\
\hline PTEN\%LOSS & $58.80 \%$ & $80.80 \%$ & 0.726 & $70.60 \%$ & $80.80 \%$ & 0.827 \\
\hline PTEN/CEP10\%LOSS & $82.40 \%$ & $80.80 \%$ & 0.881 & $64.70 \%$ & $80.80 \%$ & 0.788 \\
\hline CEP10\%Gain & $94.10 \%$ & $84.60 \%$ & 0.93 & $76.50 \%$ & $84.60 \%$ & 0.786 \\
\hline CEP10\%Abnormal & $79.40 \%$ & $80.80 \%$ & 0.865 & $76.50 \%$ & $73.10 \%$ & 0.775 \\
\hline CEP7\%Gain & $85.30 \%$ & $84.60 \%$ & 0.886 & $70.60 \%$ & $76.90 \%$ & 0.77 \\
\hline CEP8\%Abnormal & $94.10 \%$ & $88.50 \%$ & 0.966 & $64.70 \%$ & $76.90 \%$ & 0.752 \\
\hline LPL\%Abnormal & $79.40 \%$ & $92.30 \%$ & 0.945 & $64.70 \%$ & $76.90 \%$ & 0.736 \\
\hline
\end{tabular}

The analyses of tumour ROI vs. BPH, and benign ROI vs. BPH performed independently. Sensitivity and specificity calculated at the optimal cut-off in each of the two analyses for each parameter. 
highly abnormal in tumour area of adenocarcinoma RP specimens, as compared to BPH control.

The selected single FISH probe parameters listed in Table 1 were then grouped in all possible 4-probe combinations, and the ROC method was used now to identify the optimal probe combinations based on their AUC values and clinical consideration in distinguishing histologically benign ROI specimens vs. BPH. Table 2 shows the selected 4 probe combinations identified in this analysis.

As evident from Table 2, probe combination 3 had the highest AUC value of 0.938 , while probe combination 1 had the second highest AUC value (0.917) and afforded the lowest DFI (combined highest sensitivity and specificity) at the indicated cut-offs. Since the achievable combined sensitivity and specificity were noticeably higher for probe combination 1 , and since this probe combination also contained probes to two important tumourrelated loci, probe combination 1 was selected over probe combination 3 despite the somewhat higher AUC achieved with probe combination 3 . The optimal cut-off values chosen for the four individual FISH probe parameters in probe combination 1 are PTEN\%loss $>33$, CEP7\%Abnormal > 28, MYC\%gain > 35, and CEP8\%Abnormal $>34$. Using these cut-offs, probe combination 1 yielded a sensitivity of $88.2 \%$ and a specificity of $84.6 \%$ for histologically benign ROI vs. BPH $(\mathrm{AUC}=0.917$ ), while the sensitivity and specificity for tumour ROI vs. $\mathrm{BPH}$ were $100 \%$ and $84.6 \%$, respectively, with the AUC $=0.960$.

ROC curves for the selected 4- probe combination, and the corresponding 4 single FISH probe parameters, including PTEN\%loss, CEP7\%Abnormal, MYC\%gain, CEP8\%Abnormal, are plotted in Figure 3. The curves labelled 'benign ROI' were obtained from the FISH evaluation comparing the 17 histologically Benign ROI to the $26 \mathrm{BPH}$ specimens. The curve labelled 'tumour ROI' was obtained from the evaluation comparing the 33 tumour $\mathrm{ROI}$ to the $26 \mathrm{BPH}$. The corresponding AUC values for each of the plotted ROC curves are listed in the table under the ROC plot.

\section{Discussion}

Each year, millions of men are referred for prostate biopsies due to abnormal DRE or elevated serum PSA. Prostate biopsies are not only unpleasant, but also carry risks to the patient, and are expensive. Moreover, falsenegative rates for initial prostate biopsies (particularly for sextant biopsies) are routinely reported to be between $10-25 \%$, and repeat biopsies are essential components of prostate cancer detection [24]. Therefore, reliable diagnostic markers that enable early and accurate detection of prostate tumours when they are confined to the prostate are essential.

In this study, we tested $L P L, M Y C, P T E N$, CEP 7, CEP 8, and CEP $10 \mathrm{FISH}$ probes, based on the published roles of these genes, and on our initial FISH study on FFPE prostate cancer and BPH specimens. Sixteen parameters were derived from the number of FISH signals at the above loci, and compared between prostate adenocarcinoma tumour tissue (tumour ROI) and $\mathrm{BPH}$ samples by the $t$-test. In this analysis, all 16 FISH parameters demonstrated a significant difference between the comparison groups, supporting the role of these genes in prostate cancer. In addition to the tumour ROIs, we found FISH abnormalities in histologically benign tissue separated from the tumour margin on average by approximately $1 \mathrm{~cm}$ (histologically benign $\mathrm{ROI}$ ).

The pattern of FISH abnormalities in the histologically benign ROIs was similar to that found in the corresponding tumour (Additional file 3). The observed cytogenetic abnormalities appeared to be reflective of genetic changes in the cancer cells of the associated tumour, indicating the possibility that a field cancerisation effect may be manifested in prostate cancer at the cytogenetic level as a field of molecular alterations in adjacent, histologically benign areas surrounding the tumour. Genomic alterations observed outside the histologically visible

Table 2 ROC analysis of the selected four 4-probe combinations from the 10 single FISH probe parameters

\begin{tabular}{|c|c|c|c|c|c|c|c|c|}
\hline Probe combination & Probe parameters & Cut-off 1 & Cut-off 2 & Cut-off 3 & Cut-off 4 & Sensitivity & Specificity & AUC \\
\hline $\begin{array}{l}\text { Probe } \\
\text { combination } 1\end{array}$ & $\begin{array}{l}\text { PTEN\%loss, } \\
\text { CEP7\%abnormal, } \\
\text { MYC\%gain, } \\
\text { CEP8\%abnormal }\end{array}$ & 33 PTEN\% loss & $\begin{array}{l}28 \text { CEP7\% } \\
\text { abnormal }\end{array}$ & 35 MYC\% gain & $\begin{array}{l}34 \text { CEP8\% } \\
\text { abnormal }\end{array}$ & $88.20 \%$ & $84.60 \%$ & 0.917 \\
\hline $\begin{array}{l}\text { Probe } \\
\text { combination } 2\end{array}$ & $\begin{array}{l}\text { PTEN/CEP10\%loss, } \\
\text { CEP7\%abnormal, } \\
\text { MYC\%gain }\end{array}$ & 35 PTEN/CEP10\% loss & $\begin{array}{l}25 \text { CEP7\% } \\
\text { abnormal }\end{array}$ & 35 MYC\% gain & NA & $82.40 \%$ & $80.80 \%$ & 0.911 \\
\hline $\begin{array}{l}\text { Probe } \\
\text { combination } 3\end{array}$ & $\begin{array}{l}\text { PTEN/CEP10\%loss, } \\
\text { CEP7\%abnormal, } \\
\text { CEP8\%abnormal }\end{array}$ & 35 PTEN/CEP10\% loss & $\begin{array}{l}25 \text { CEP7\% } \\
\text { abnormal }\end{array}$ & $\begin{array}{l}34 \text { CEP8\% } \\
\text { abnormal }\end{array}$ & NA & $88.20 \%$ & $80.80 \%$ & 0.938 \\
\hline $\begin{array}{l}\text { Probe } \\
\text { combination } 4\end{array}$ & $\begin{array}{l}\text { PTEN/CEP10\%loss, } \\
\text { MYC\%gain, CEP8gain }\end{array}$ & 35 PTEN/CEP10\% loss & 5 MYC\% gain & 35 CEP8\% gain & NA & $64.70 \%$ & $88.50 \%$ & 0.834 \\
\hline
\end{tabular}

Cut-off 1, cut-off 2, cut-off 3, and cut-off 4 are the optimal probe cut-offs (providing lowest DFI), respectively. 


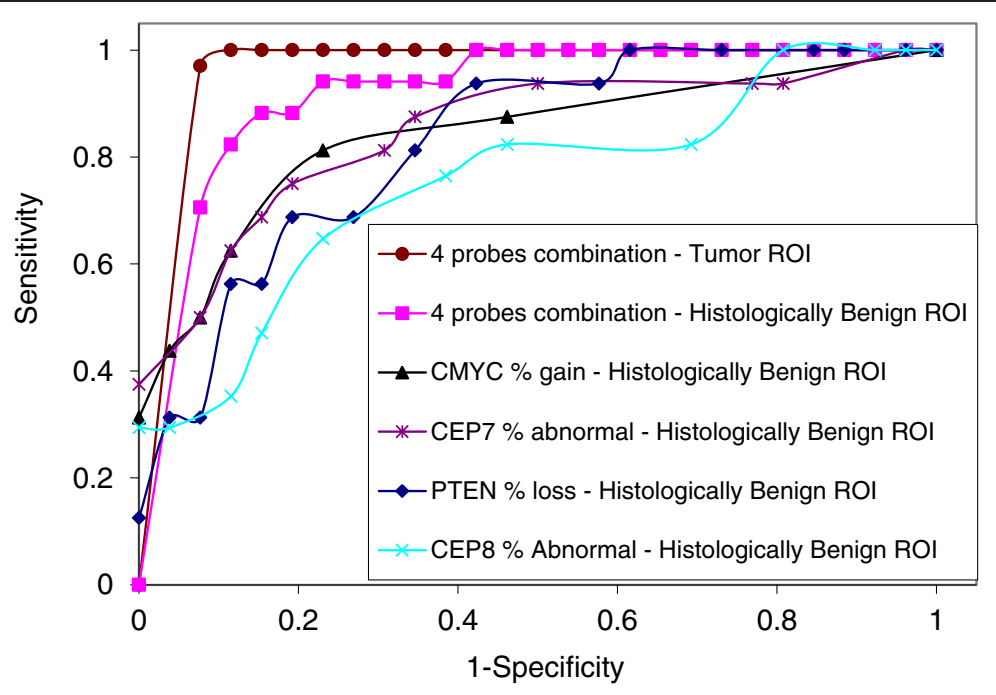

\begin{tabular}{|l|l|}
\hline \multicolumn{1}{|c|}{ Parameter } & AUC \\
\hline 4 Probes Combination-Tumour ROI & 0.960 \\
\hline 4 Probes Combination - Histologically Benign ROI & 0.917 \\
MYC\%Gain - Histologically Benign ROI & 0.846 \\
CEP7\%Abnormal - Histologically Benign ROI & 0.845 \\
PTEN\%Loss - Histologically Benign ROI & 0.827 \\
CEP8\%Abnormal - Histologically Benign ROI & 0.752 \\
\hline
\end{tabular}

Figure 3 ROC curve. ROC plot for individual FISH probe parameters (PTEN\%loss, CEP7\%abnorm, MYC\%gain, CEP8\%abnormal) and the 4-probe combination. Data were calculated from the FISH evaluation of the 17 benign ROI (histologically benign regions surrounding the tumour) and 26 $\mathrm{BPH}$ specimens. The ROC plot for the 4-probe combination of the 33 tumour ROI and $26 \mathrm{BPH}$ specimens are also shown here. The AUC of the ROC curves are shown in the table.

tumour margins could result from pre-existing fields of precursor cells in which cancer develops; alternatively, the tumour could have an effect on the surrounding tissue, or the observed abnormalities could reflect both of the above abnormalities [15]. We also cannot rule out that the cells in which genomic abnormalities were detected represent events related to the metastatic tumour spread, such as undetected micro-metastases. However, although FISH does not have an ability to establish whether a cell under interrogation is a tumour cell, a precursor cancer cell or a benign cell, cytogenetic abnormalities detected by FISH in our study were similar to those in the associated tumour.

Not all chromosomal loci were detectable in the field surrounding the tumour at the same level. Out of the initial sixteen FISH parameters chosen for evaluation, 10 were identified that detected FISH abnormalities in histologically benign ROIs of RP adenocarcinoma specimens, specifically CEP10\%Abnormal, CEP10\%Gain, CEP7\%Abnormal, CEP7\%Gain, CEP8\%Abnormal, CEP8\%Gain, MYC\%Gain, LPL\%Abnormal, PTEN\%Loss, PTEN/CEP10\%Loss.
By combining the individual FISH parameters, we identified a probe combination (PTEN, MYC, CEP7 and CEP8) that was superior in performance to that of individual probes (Table 2 and Figure 3). In the specimen set used in this study, with the optimal cut-offs for each FISH parameter selected using the ROC method, the sensitivity of the 4-probe combination was $88.2 \%$ and the specificity was $84.6 \%$ for discriminating prostate adenocarcinoma from BPH specimens based on cytogenetic abnormalities found in histologically benign regions surrounding the tumour. Using the same cut-offs for each FISH parameter, a sensitivity of $100 \%$ and a specificity of $84.6 \%$ were achieved for discriminating prostate adenocarcinoma from $\mathrm{BPH}$ specimens based on cytogenetic abnormalities found within the tumour. These findings support the field cancerisation effect of prostate cancer reported in several studies, including some of the recent work of methylation changes [25], Telomere attrition [26], Mitochondrial DNA changes [27], and Gene expression changes [28,29]. Significantly, evidence for such malignancy-associated changes has 
been presented in other organs such as the cervix, bladder and breast [11].

The results of our feasibility study of radical prostatectomy suggest that multi-colour FISH may find utility in detecting cytogenetic abnormalities associated with adenocarcinoma of prostate in the field around the tumour, and therefore could potentially aid in assessing negative biopsies of patients with suspected cancer. The hypothesis that FISH could be used to aid the detection of adenocarcinoma by assessing the tissue surrounding the tumour will be validated in the next phase of the investigation using prostate needle biopsy specimens.

\section{Conclusions}

In this study of radical prostatectomy specimens, cytogenetic abnormalities were observed by FISH within regions of prostate adenocarcinoma, as well as within regions of benign histology extending beyond histologically evident tumour margin, indicating a field cancerisation effect in prostate cancer. Detection of field cancerisation by FISH may prove to have a utility in the evaluation of histologynegative biopsies from patients suspected of having prostate cancer, and therefore could aid histopathological evaluation by providing an indication of possible presence of malignancy. Although preliminary, the findings of a FISH panel with sensitivity $>85 \%$ in histologically benign regions away from tumour and specificity $~ 85 \%$ in $\mathrm{BPH}$, provide encouragement to pursue the utility of these cytogenetic markers further. Validation in a larger cohort of patients with both positive and negative biopsies is envisioned to confirm our findings.

\section{Additional files}

Additional file 1: H\&E Images of the 5 - $\mu \mathrm{m}$ sections of the 17 histologically benign specimens from the corresponding radical prostatectomy adenocarcinoma cases. For each case, the specimen slide used for the FISH analysis was within 10 serial sections of the H\&E stained slide.

Additional file 2: $t$-test analyses. Table S2a. $t$-test of tumour ROI vs BPH. Table S2b. $t$-test of histologically benign $\mathrm{ROI}$ vs $\mathrm{BPH}$.

Additional file 3: The pattern of FISH abnormalities presents in the 33 tumour ROls and the 17 available corresponding histologically benign ROIs.

\section{Abbreviations \\ AUC: Area under the curve; BPH: Benign prostatic hyperplasia; CaP: Prostate cancer; CEP: Centromeric probes; DRE: Digital rectal examination; FFPE: Formalin fixed paraffin embedded; FISH: Fluorescence in situ hybridisation; LSI: Locus specific identifier; PSA: Prostate-specific antigen; ROC: Receiving operating characteristic; RP: Radical prostatectomy; ROI: Region of interest.}

\section{Competing interests}

Authors $Y Z, K P$, LM have filed a pending patent application relating to the subject matter of this article, which patent application has been assigned to Abbott Molecular Inc.

\section{Authors' contributions}

YZ analysed the histological samples, performed the statistical analysis, and drafted the manuscript. TP analysed the histological samples. BB analysed the histological samples. JD performed the statistical analysis and helped to draft the statistical section. PL performed the statistical analysis and helped to draft the statistical section. DE assisted in the appropriate specimen selection and sectioned the slides. JC provided clinical opinion, selected and provided appropriate specimens and helped conceive the study, and scribed the tumour region on the H\&E slides. LM conceived the study and the study design, provided guidance on data analysis, KP coordinated the study and its design, analysed the histological samples, assisted in selection of probes, performed the statistical analysis, and helped draft the manuscript. All authors read and approved the final manuscript.

\section{Acknowledgements}

We thank John Schulz and Mona Legator (Abbott Molecular R\&D) for designing and manufacturing FISH probes for this study. We gratefully acknowledge Dr. Klara Abravaya, Sr. Director of Abbott Molecular R\&D, for sponsoring this study and for the review of this manuscript. We also thank Dr. Tracey Colpitts, Director of Abbott Molecular Technology Assessment for helping to prepare response to the reviewers of this manuscript. We thank Frank Policht for helping to take images.

\section{Author details}

${ }^{1}$ Abbott Molecular, Inc, 1300 East Touhy Avenue, Des Plaines, IL 60018, USA. ${ }^{2}$ Department of Pathology, Rush University Medical Center, 1750 West Harrison Street, Chicago, IL 60612, USA.

Received: 1 August 2013 Accepted: 12 February 2014

Published: 25 February 2014

\section{References}

1. Siegel R, Naishadham D, Jemal A: Cancer statistics. CA Cancer J Clin 2012, 62:10-29.

2. Shariat SF, Roehrborn CG: Using biopsy to detect prostate cancer. Rev Urol 2008, 10(4):262-280.

3. Nonn L, Ananthanarayanan V, Gann PH: Evidence for field cancerization of the prostate. Prostate 2009, 69:1470-1479.

4. Campos-Fernandes JL, Bastien L, Nicolaiew N, Robert G, Terry S, Vacherot F, Salomon L, Allory Y, Vordos D, Hoznek A, Yiou R, Patard JJ, Abbou CC, de la Taille A: Prostate cancer detection rate in patients with repeated extended 21-sample needle biopsy. Eur Urol 2009, 55:600-609.

5. Boccon-Gibod LM, Dumonceau O, Toublanc M, Ravery V, Boccon-Gibod LA: Micro-focal prostate cancer: a comparison of biopsy and radical prostatectomy specimen features. Eur Urol 2005, 48:895-899.

6. Arora R, Koch MO, Eble JN, Ulbright TM, Li L, Cheng L: Heterogeneity of gleason grade in multifocal adenocarcinoma of the prostate. Cancer 2004, 100:2362-2366.

7. Qian J, Wollan P, Bostwick DG: The extent and multicentricity of High-grade prostatic intraepithelial neoplasia in clinically localized prostate adenocarcinoma. Hum Pathol 1997, 28:143-148.

8. de la Taille A, Antiphon P, Salomon L, Cherfan M, Porcher R, Hoznek A, Saint F, Vordos D, Cicco A, Yiou R, Zafrani ES, Chopin D, Abbou CC: Prospective evaluation of a 21-sample needle biopsy procedure designed to improve the prostate cancer detection rate. Urology 2003, 61(6):1181-1186.

9. Mohler J, Bahnson RR, Boston B, Busby JE, D'Amico A, Eastham JA, Enke CA, George D, Horwitz EM, Huben RP, Kantoff P, Kawachi M, Kuettel M, Lange PH, Macvicar G, Plimack ER, Pow-Sang JM, Roach M 3rd, Rohren E, Roth BJ, Shrieve DC, Smith MR, Srinivas S, Twardowski P, Walsh PC: NCCN clinical practice guidelines in oncology: prostate cancer. J Natl Compr Canc Netw 2010, 8:162-200.

10. Slaughter DP, Southwick HW, Smejkal W: Field cancerization in oral stratified squamous epithelium; clinical implications of multicentric origin. Cancer 1953, 6:963-968.

11. Höckel M, Dornhöfer $N$ : The hydra phenomenon of cancer: why tumors recur locally after microscopically complete resection. Canc Res 2005, 65(8):2997-3002

12. Chai $H$, Brown RE: Review: field effect in cancer-an update. Ann Clin Lab Sci 2009, 39(4):331-337.

13. Risk MC, Knudsen BS, Coleman I, Dumpit RF, Kristal AR, LeMeur N, Gentleman RC, True LD, Nelson PS, Lin DW: Differential gene expression in 
benign prostate epithelium of Men with and without prostate cancer: evidence for a prostate cancer field effect. Clin Canc Res 2010, 16:5414-5423.

14. Trujillo KA, Jones AC, Griffith JK, Bisoffi M: Markers of field cancerization: proposed clinical applications in prostate biopsies. Prostate Canc 2012, 2012:302894.

15. Haaland CM, Heaphy CM, Butler KS, Fischer EG, Griffith JK, Bisoffi M: Differential gene expression in tumoradjacent histologically normal prostatic tissue indicates field cancerisation. Int J Oncol 2009, 35:537-546.

16. Bishop R: Applications of fluorescence in situ hybridization (FISH) in detecting genetic aberrations of medical significance. Biosci Horiz 2010, 3(1):85-95.

17. Dellas A, Torhorst J, Jiang F, Proffitt J, Schultheiss E, Holzgreve W, Sauter G, Mihatsch MJ, Moch H: Prognostic value of genomic alterations in invasive cervical squamous cell carcinoma of clinical stage IB detected by comparative genomic hybridization. Canc Res 1999, 59(14):3475-3479.

18. Makarov DV, Loeb S, Getzenberg RH, Partin AW: Biomarkers for prostate cancer. Annu Rev Med 2009, 60:139-151.

19. Nelson WG, De Marzo AM, Isaacs WB: Mechanisms of disease prostate cancer. N Engl J Med 2003, 349:366-381.

20. Al-Maghrabi J, Vorobyova L, Toi A, Chapman W, Zielenska M, Squire JA: Identification of numerical chromosomal changes detected by interphase fluorescence in situ hybridization in high-grade prostate intraepithelial neoplasia as a predictor of carcinoma. Arch Pathol Lab Med 2002, 126(2):165-169.

21. Bova GS, Carter BS, Bussemakers MJ, Emi M, Fujiwara Y, Kyprianou N, Jacobs SC, Robinson JC, Epstein JI, Walsh PC, Isaacs WB: Homozygous deletion and frequent allelic loss of chromosome $8 \mathrm{p} 22$ loci in human prostate cancer. Cancer Res 1993, 53:3869-3873.

22. Yoshimoto M, Cunha IW, Coudry RA, Fonseca FP, Torres CH, Soares FA, Squire JA: FISH analysis of 107 prostate cancers shows that PTEN genomic deletion is associated with poor clinical outcome. $\mathrm{Br} J$ Cancer 2007, 97(5):678-685. Epub 2007 Aug 14.

23. Metz CE: Receiver operating characteristic analysis: a tool for the quantitative evaluation of observer performance and imaging systems. J Am Coll Radiol 2006, 3:413-422.

24. Yanke BV, Gonen M, Scardino PT, Kattan MW: Validation of a nomogram for predicting positive repeat biopsy for prostate cancer. J Urol 2005, $173: 421-424$

25. Mehrotra J, Varde S, Wang H, Chiu H, Vargo J, Gray K, Nagle RB, Neri JR, Mazumder A: Quantitative, spatial resolution of the epigenetic field effect in prostate cancer. Prostate 2008, 68(2):152-160

26. Joshua AM, Shen E, Yoshimoto M, Marrano P, Zielenska M, Evans AJ, Van der Kwast T, Squire JA: Topographical analysis of telomere length and correlation with genomic instability in whole mount prostatectomies. Prostate 2011, 71(7):778-790.

27. Reguly B, Jakupciak JP, Parr RL: 3.4 kb mitochondrial genome deletion serves as a surrogate predictive biomarker for prostate cancer in histopathologically benign biopsy cores. J Canadian Urolog Assoc 2010, 4(5):E118-E122.

28. Mazzucchelli R, Barbisan F, Santinelli A, Lopez-Beltran A, Cheng L, Scarpelli M, Montironi R: Immunohistochemical expression of prostate tumor overexpressed 1 in cystoprostatectomies with incidental and insignificant prostate cancer. Further evidence for field effect in prostatic carcinogenesis. Hum Pathol 2011, 42(12):1931-1936. doi:10.1016/j.humpath.2011.02.014. Epub 2011 Jun 14.

29. Jones AC, Trujillo KA, Phillips GK, Fleet TM, Murton JK, Severns V, Shah SK, Davis MS, Smith AY, Griffith JK, Fischer EG, Bisoffi M: Early growth response 1 and fatty acid synthase expression is altered in tumor adjacent prostate tissue and indicates field cancerization. Prostate 2012 72(11):1159-1170. doi:10.1002/pros.22465. Epub 2011 Nov 29.

doi:10.1186/1471-2407-14-129

Cite this article as: Zhang et al:: Identification of FISH biomarkers to detect chromosome abnormalities associated with prostate adenocarcinoma in tumour and field effect environment. BMC Cancer 2014 14:129

\section{Submit your next manuscript to BioMed Central and take full advantage of:}

- Convenient online submission

- Thorough peer review

- No space constraints or color figure charges

- Immediate publication on acceptance

- Inclusion in PubMed, CAS, Scopus and Google Scholar

- Research which is freely available for redistribution

Submit your manuscript at www.biomedcentral.com/submit
C Biomed Central 